\section{Circulating potential}

The innate lymphoid cell (ILC) group, composed of helper (ILC1, ILC2 and ILC3) and cytotoxic subsets (NK cells), arises from ILC-committed precursors (ILCPs). In Cell, Di Santo and colleagues identify CD117+ ILCPs at low frequency in human peripheral blood (PB). This population lacks expression of signature transcription factor genes associated with the various ILC subsets; instead these genes are in an epigenetically poised state. In vitro stimulation with defined cytokine cocktails or clonal analysis after culture on OP9 cells shows that the PB ILCPs have the potential to give rise to any of the ILC subsets in a uni- and/or multipotent manner but have no $\mathrm{T}$ or $\mathrm{B}$ cell potential. Similarly, immune-deficient mice 'humanized' with CD117+ PB ILCPs generate ILC populations that include NK cells in various tissues-lymphoid and non-lymphoid. Humans therefore possess a circulating ILC precursor population that can seed peripheral tissues and, with the appropriate environmental cues, give rise to all ILC populations.

Cell (9 March 2017) doi:10.1016/j.cell.2017.02.021

\section{Vitamin C for microglia}

High concentrations of vitamin $\mathrm{C}$ are found in the central nervous system. In Science Signaling, Portugal et al. show that brain-resident microglia require vitamin $\mathrm{C}$ for homeostasis. Microglia import extracellular ascorbate, the reduced form of vitamin C, via sodium-vitamin C cotransporter 2 (SVCT2). Intracellular ascorbate reduces activation and nuclear translocation of the transcription factor NF- $\mathrm{BB}$, thereby limiting the production of proinflammatory mediators. Conversely, exposure to lipopolysaccharide activates microglia by inducing SVCT2 internalization and lysosomal degradation by a mechanism that involves Src kinase and caveolin-1. Reduced expression or inhibition of SVCT2 also activates microglia, thus leading to increased expression of tumor necrosis factor, interleukin 6 (IL-6), IL-1 $\beta$, MHC class II molecules, TREM2, iNOS and inflammatory chemokines. The question of whether loss of SVCT2 is involved in neuroinflammatory disease deserves further attention.

$L A D$

Sci. Signal. 10, eaal2005 (2017)

\section{Macrophage dynamics}

Exposure to unmethylated CpG DNA reduces the risk of asthma in mice and humans. In Immunity, Bureau and colleagues show that $\mathrm{CpG}$ expands a population of lung interstitial macrophages previously known to constitutively produce interleukin 10 (IL10) and to protect mice from asthma. Interstitial macrophages are $\mathrm{Ly} 6 \mathrm{C}^{\mathrm{lo}} \mathrm{CD} 64^{\mathrm{lo}}$ and produce IL-10 after stimulation with CpG and lipopolysaccharide. CpG protects mice from asthma induced by sensitization with various agents, and interstitial macrophages from $\mathrm{CpG}$-treated mice can transfer protection in a manner dependent on IL-10. At steady-state, these interstitial macrophages are partially maintained by CCR2-dependent bloodderived monocytes. However, their CpG-induced expansion is independent of local proliferation, sustained by recruitment of monocytes from the spleen and independent of CCR2, CCL2, CCL5 and CCL9.

\section{Marking HIV}

The persistence of the HIV reservoir is a major obstacle to potential cures. In Nature, Benkirane and colleagues show that upregulation of CD32a, the low-affinity IgG receptor, is specific for HIV-1-infected quiescent $\mathrm{CD}^{+} \mathrm{T}$ cells. The authors create an experimental model of infection of resting $\mathrm{CD}^{+}{ }^{+} \mathrm{T}$ cells, which are usually nonpermissive to HIV-1, by transfecting peripheral blood mononuclear cells with the SIV accessory protein Vpx, which degrades the restriction factor SAMHD1. Among the 16 transmembrane-protein-encoding genes that are specifically upregulated by HIV-1 infection in resting CD $4^{+}$T cells, FCGR2A (encoding CD32a) is upregulated 50 -fold compared with its expression in non-infected cells. CD32a is not induced by productive infection with HIV-1 in proliferating $\mathrm{CD}^{+} \mathrm{T}$ cells. In $12 \mathrm{HIV}$-1-infected individuals undergoing antiretroviral therapy, $\mathrm{CD} 32 \mathrm{a}^{\text {hi }} \mathrm{CD} 4^{+} \mathrm{T}$ cells were found to harbor up to three copies of HIV-1 DNA per cell, make up $26-86 \%$ of the $\mathrm{CD} 4^{+} \mathrm{T}$ cell viral reservoir, and harbor replication-competent proviruses. Thus, CD32a marks CD $4^{+}$ $T$ cells in the viral reservoir.

Nature 543, 564-567 (2017)

\section{PD-1 targets CD28}

The coinhibitory receptor PD-1 is an important target of immunotherapy now used in the clinic, yet exactly how PD-1 inhibits T cell function remains unclear. In Science, Kamphorst et al. and Hui et al. identify the T cell costimulatory receptor CD28 as the relevant target of PD-1-mediated inhibition. The former study shows that CD28 costimulation is required for $\mathrm{CD}^{+} \mathrm{T}$ cell proliferation after PD-1 blockade in mice, using chronic viral infection and tumor models. Preferential proliferation of CD28 ${ }^{+} \mathrm{T}$ cells is also seen in patients receiving anti-PD-1 therapies. Hui et al. show that PD-1 is phosphorylated by the kinase Lck upon PD-L1 ligation. This interaction brings PD-1 and CD28 into proximity. Phosphorylated PD-1 specifically recruits the phosphatase Shp2, which then dephosphorylates CD28 to terminate its signaling cascade and thereby inhibit $T$ cell proliferation. These findings lend insights into how costimulatory molecules can be manipulated to enhance or inhibit adaptive immunity.

Science (9 March 2017) doi:10.1126/science.aaf0683 \& doi: $10.1126 /$ science.aaf 1292

\section{Glioma immune evasion}

Specific gain-of-function mutations in the enzyme IDH1 (IDH-MUT) frequently occur early in human glioma. In the Journal of Clinical Investigation, Okada and colleagues investigate the functional effects of IDH-MUT using human glioma samples and a novel orthotopic mouse model. The presence of IDH-MUT in human glioma is associated with lower numbers of infiltrating cytotoxic T lymphocytes (CTLs), as well as reduced expression of mRNA encoding effector molecules (e.g., GZMA) and the T cell homing chemokines CXCL9 and CXCL10. Mouse glioma cell lines transfected with IDH-MUT and implanted in recipients show similar reductions in CTL signature. The presence of IDH-MUT reduces glioma production of CXCL9 and CXCL10 at least in part through the loss of the transcription factor STAT1. Specific inhibition of IDH-MUT improved the efficacy of therapeutic peptide vaccination in the orthotopic mouse model of glioma. Targeting of mutant IDH1 activity may therefore be a useful approach in the treatment of glioma.

J. Clin. Invest. (20 March 2017) doi:10.1172/JCI90644 